\title{
Chitosan crosslinking into magnetic multi-walled carbon nanotubes beads for efficient $\mathrm{Cr}(\mathrm{VI})$ removal
}

Mian Muhammad Ahson Aslam, PhD candidate

Hsion-Wen Kuo, Associate Professor

Department of Environmental Science and Engineering

Tunghai University, Taichung, Taiwan

Walter Den, Professor of Water Resources Science and Technology

Texas A\&M University-San Antonio, TX 


\section{Heavy Metals Adsorption}

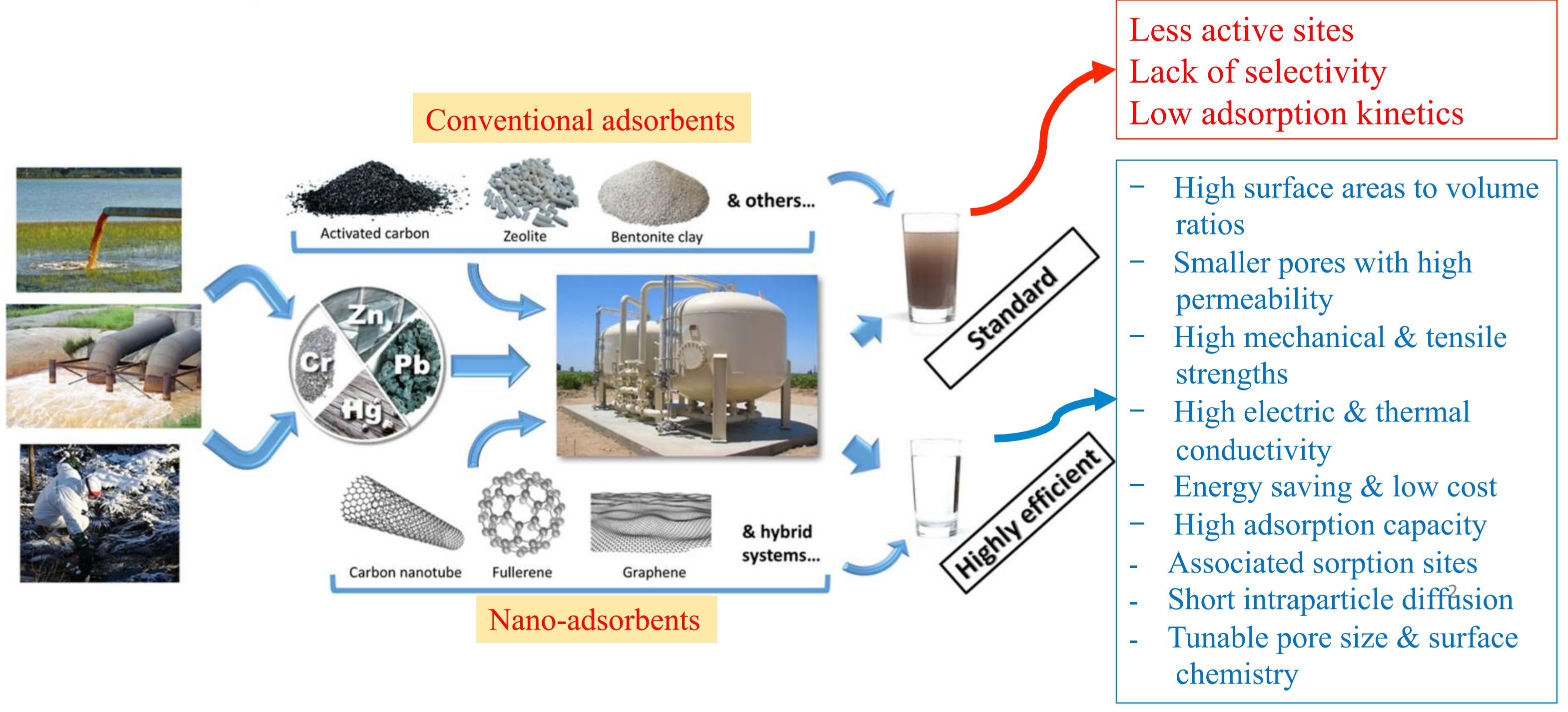




\section{Carbon Nanotubes}

As grown CNTs

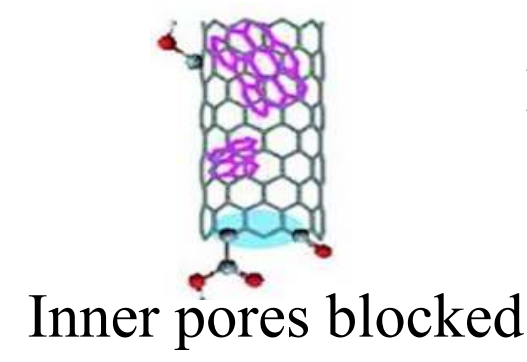

$$
\text { Heat treated } \sim 350^{\circ} \mathrm{C}
$$

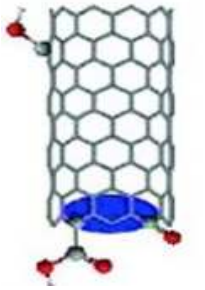

Amorphous carbon removed, metal catalyst oxidized, adsorption increased

\section{Catalyst}

Oxidized catalyst

88 Amorphous carbon
Functionalized

Acid treatment

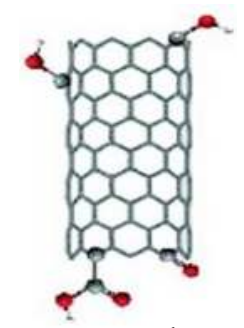

Catalyst removed, adsorption increased

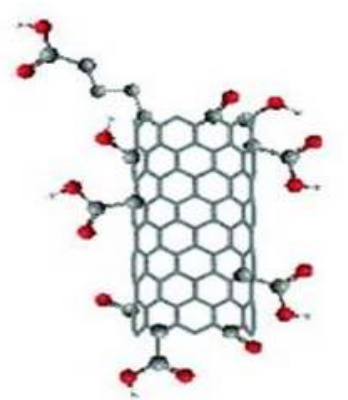

New functional groups added For nonpolar/planer chemical: adsorption decreases and for polar chemical: adsorption increased
Non-covalent functionalization

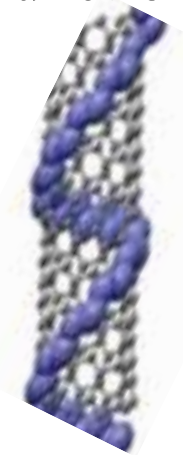

Wrapping the polymers as additional functional groups such as chitin/chitosan can further increases the adsorption

Pan and Xing, B. (2008). Environ. Sci. Technol. 42, 9005-9013. Gatabi et al. (2016). J. Molecul. Liq. 216, 117-125. 


\section{Chitosan}

- Chitosan is an excellent bio-adsorbent due to high hydrophilicity, amine and hydroxyl groups.

- It has been applied in the treatment of organic pollutants, including HMs in aqueous environmental system.

- Previously demonstrated that the adsorption capacity of CNTs can be further enhance via modifications with chitosan.

Gatabi et al. (2016). J. Molecul. Liq. 216, 117-125. Huang, et al. (2018). Chem. Eng. J. 339, 259-267.

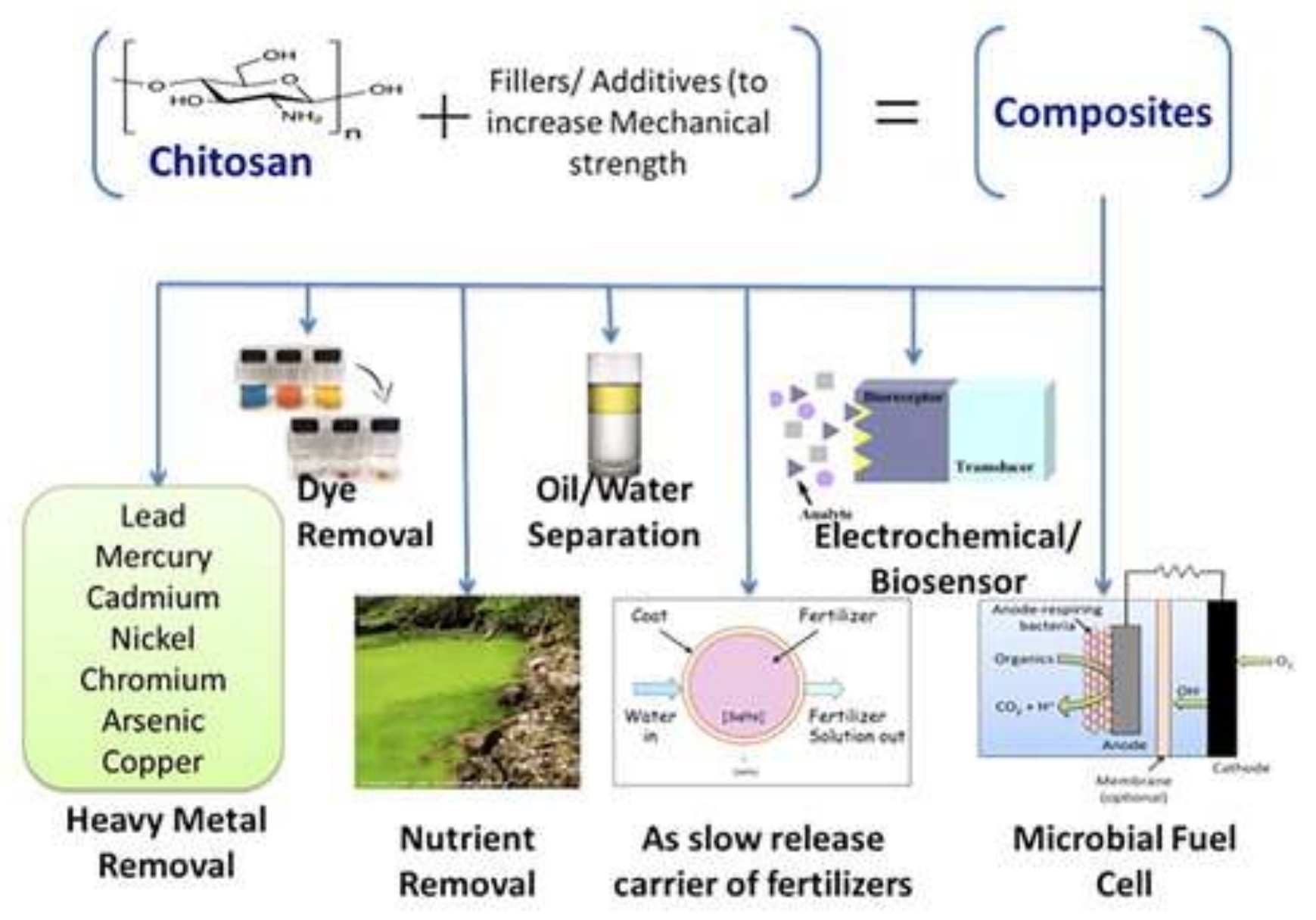




\section{Encapsulation}

- Alginate has been utilized for the encapsulation of chemical and biological compounds due to its nontoxicity, high biocompatibility, and especially, the ability to form crosslinks with cations for the material stabilization

- Surface functional groups of alginate are able to capture metallic ions via ion exchange
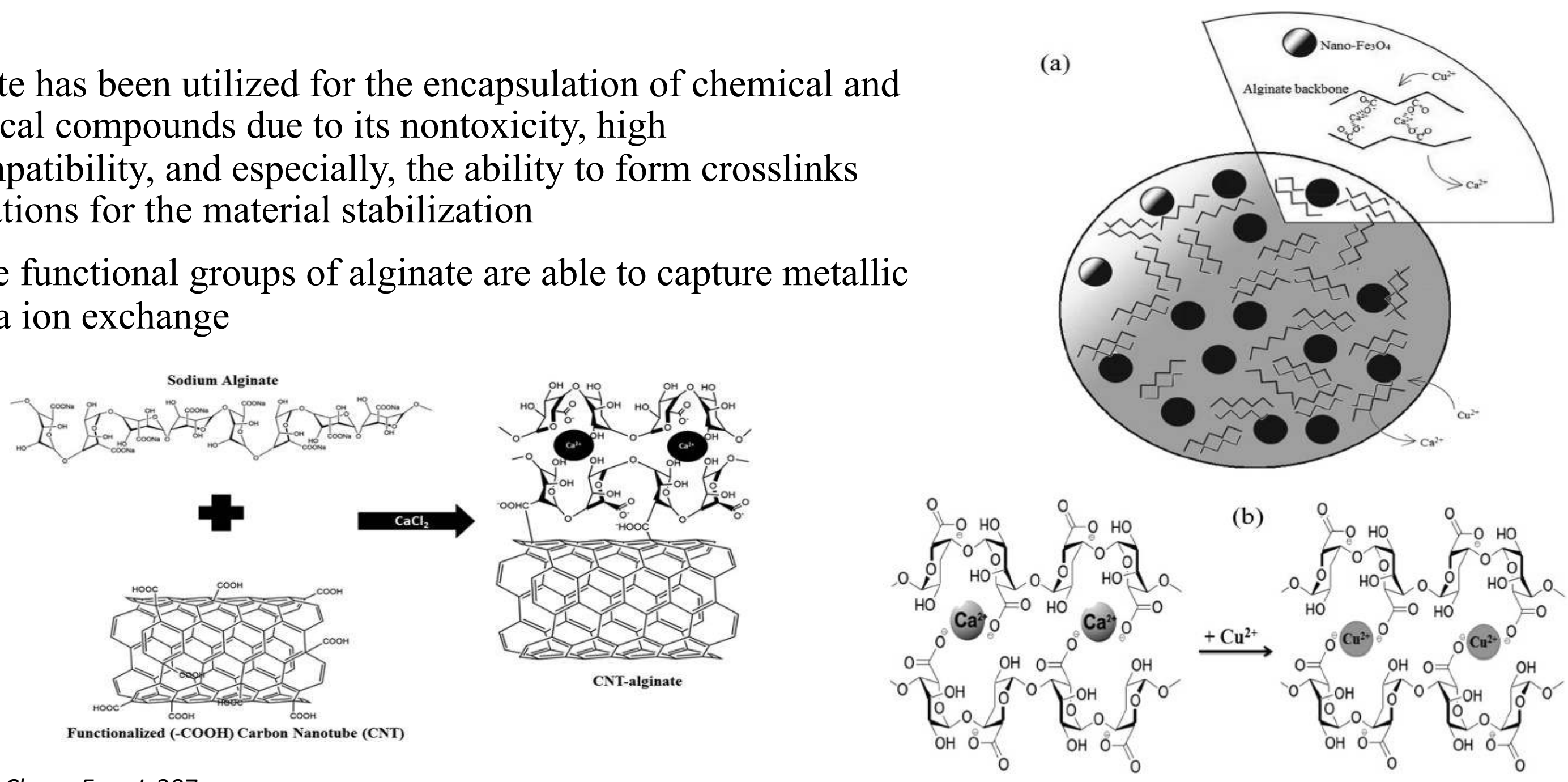


\section{Objectives}

- Developing CS/MWCNTs/Fe beads to enhance adsorptive performance for eliminating $\mathrm{Cr}(\mathrm{VI})$ from water.

- Verifying the synergistic effects between CNTs with functionalized surface and CS with rich amine groups, aided by iron particles for magnetism and facilitation of electron transfer.

- Investigating the effects of parameters i.e. adsorbent dosage, contact time, reaction temperature, solution $\mathrm{pH}$, initial concentration of metals ions.

- Determining adsorption isotherm, kinetics and thermodynamics characteristics.

- Development of a suitable regeneration scheme to make cost-effective sorbent, and determination of the potential of reusability of CS/MWCNTs/Fe beads for consecutive treatment cycles. 


\section{Material Synthesis}

\section{CS/MWCNTs/Fe beads}

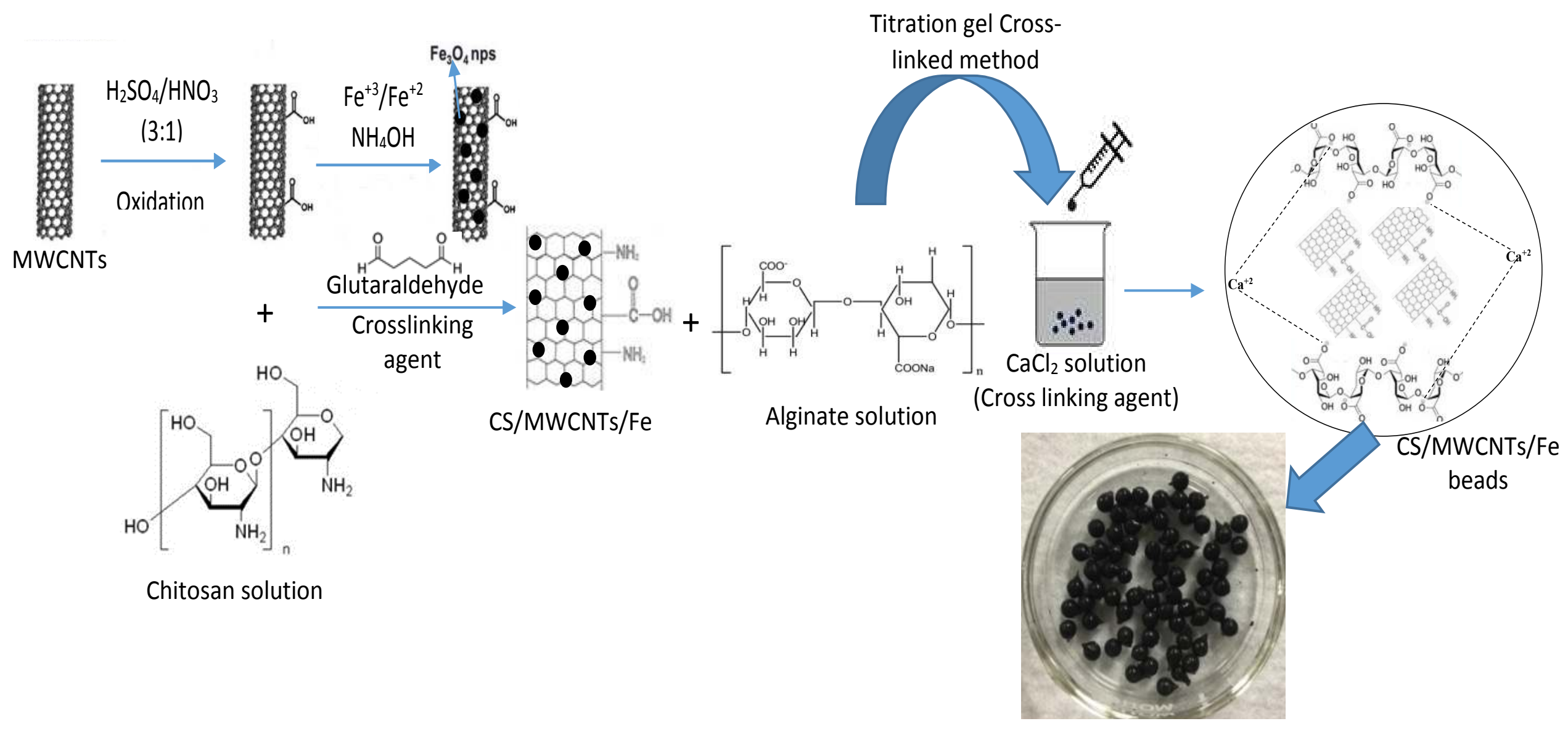




\section{Materials Characterization}

- Morphological study

- FE-SEM (JEOL JSM 700)

- Energy Dispersive Spectrometry

- Surface chemical analysis

- Fourier-transform infrared spectroscopy (FTIR) IR (JASCO FT/IR-460 Plus)

- Chrystallinity analysis

- X-ray diffractometry (XRD) (PHILIPS X'PERT Pro MPD)

- Thermal stability analysis

- Thermogravimetric analyzer (TGA) 


\section{Experimental Design}

Batch adsorption experiments

- Operating parameters such as adsorbent dosage, contact time, adsorbate concentrations, temperature and $\mathrm{pH}$ were evaluated

- $\mathrm{Cr}(\mathrm{VI})$ by UV-vis spectrophotometer

- Total chromium was measured by ICP-MS

- Optimization studies were conducted by varying the contents of MWCNTs, magnetic particles, chitosan and sodium alginate

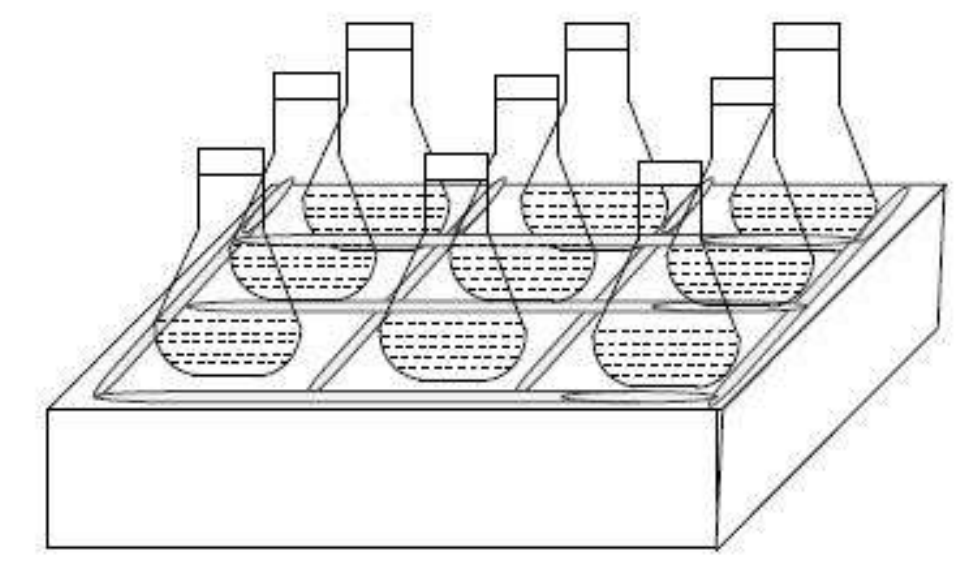

- Different adsorption isotherms and kinetic models were applied to fit the experimental data

- Thermodynamic studies were conducted at varying temperature 


\section{Morphology (FE-SEM)}

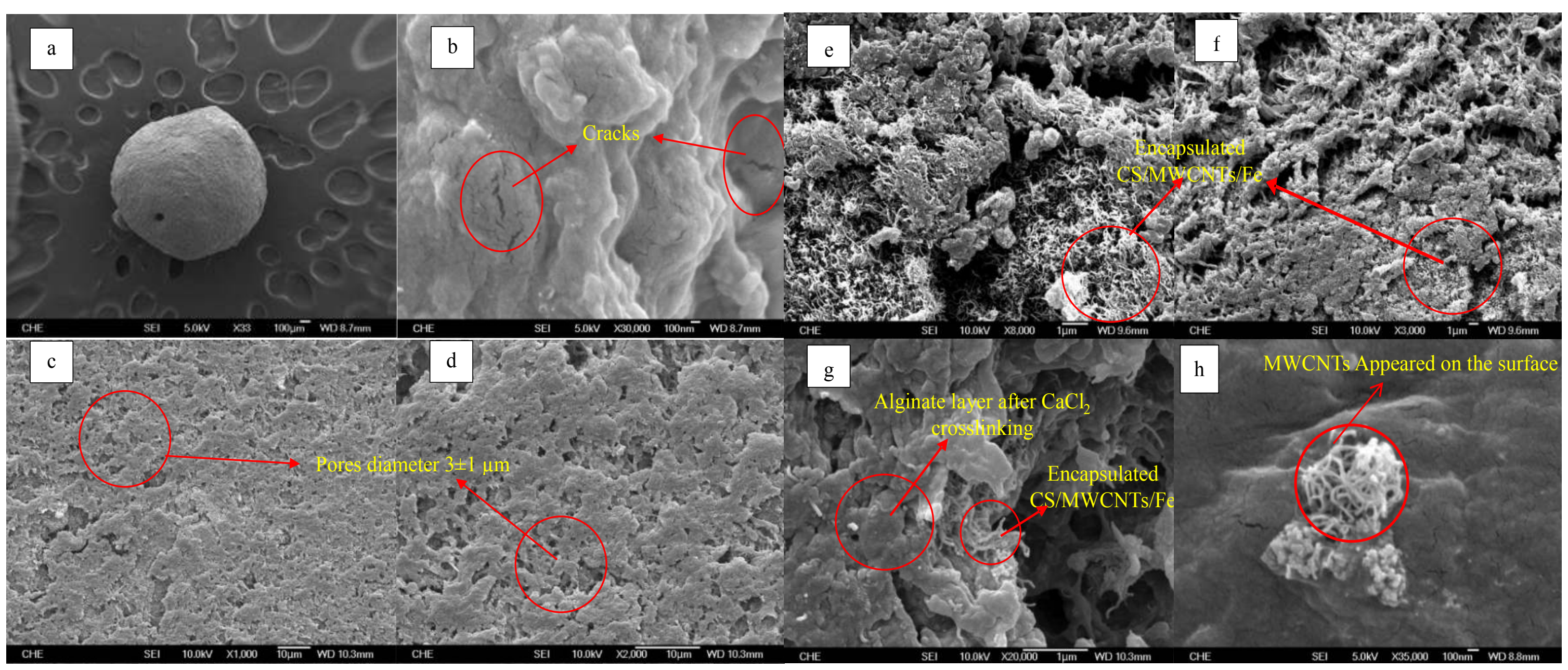




\section{Chemical Analyses}

\section{Elemental Composition (EDS)}

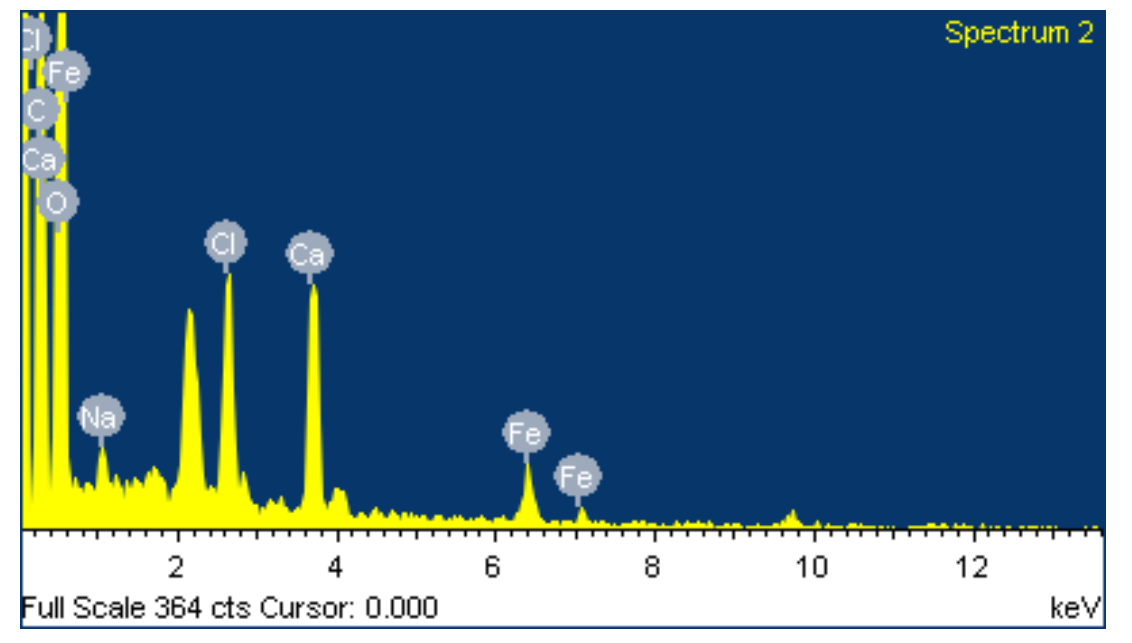

\section{Element Weight\% Atomic\%}

\begin{tabular}{rrr}
\hline $\mathrm{C}$ & 35.36 & 46.23 \\
$\mathrm{O}$ & 48.27 & 47.39 \\
$\mathrm{Na}$ & 0.86 & 0.59 \\
$\mathrm{Cl}$ & 4.48 & 1.98 \\
$\mathrm{Ca}$ & 6.37 & 2.50 \\
$\mathrm{Fe}$ & 4.66 & 1.31 \\
\hline
\end{tabular}

\section{FTIR spectrum}

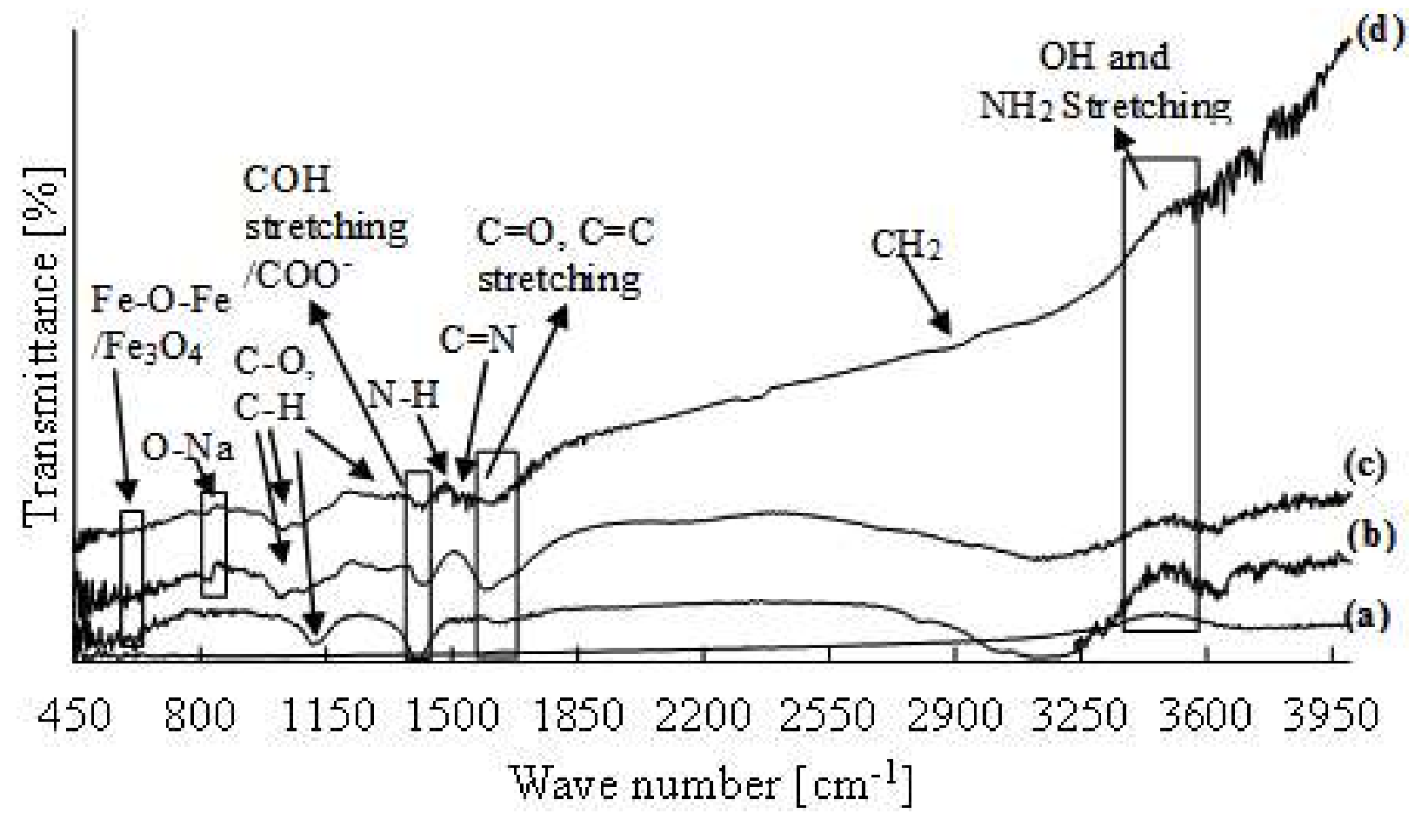
(a) Oxidized-MWCNTs
(b) $\mathrm{MWCNTs} / \mathrm{Fe}$
(c) $\mathrm{MWCNTs} / \mathrm{Fe}$ beads
(d) CS/MWCNTs/Fe beads 


\section{Characterizations}

\section{XRD analysis}

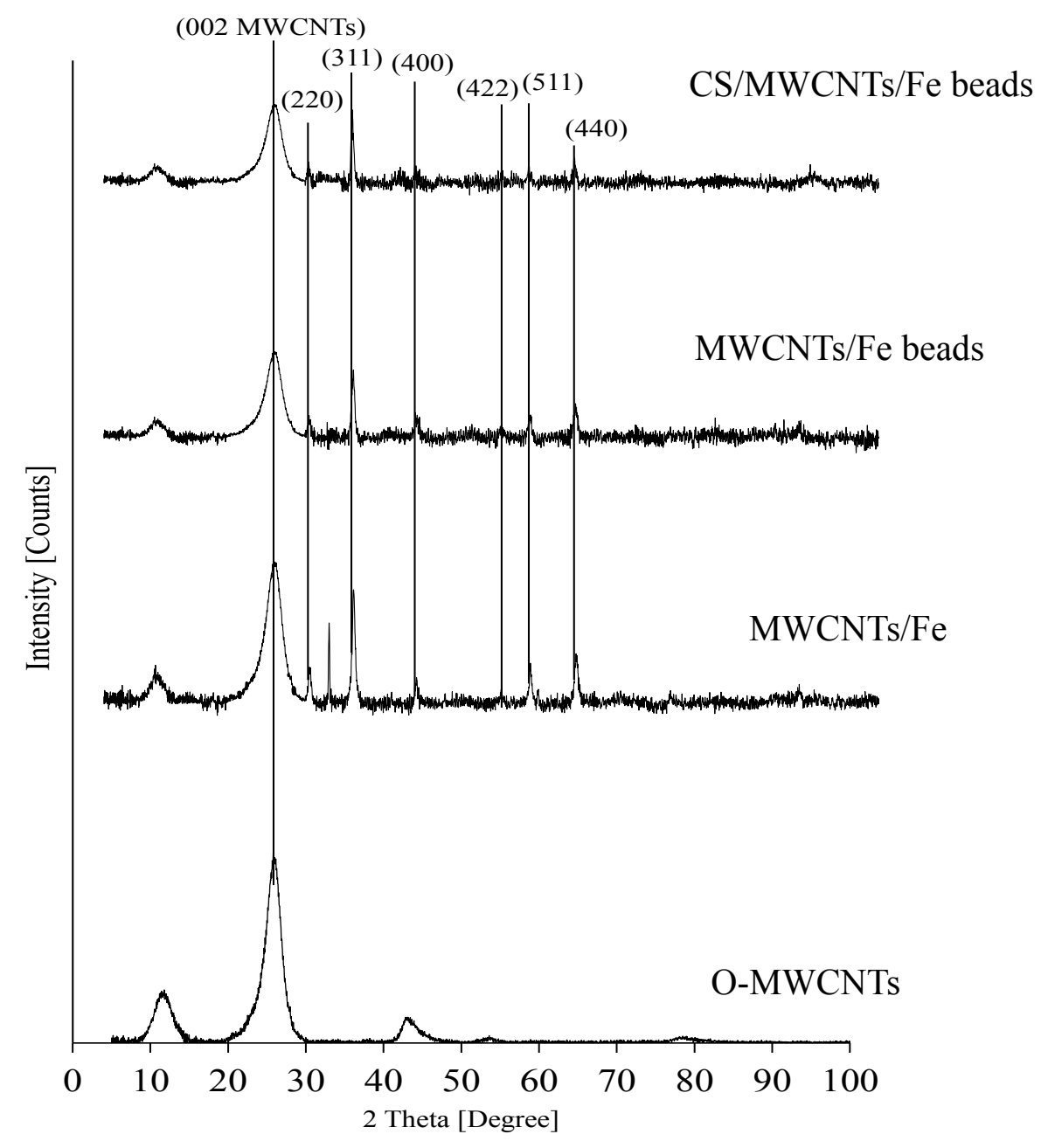

\section{TGA}

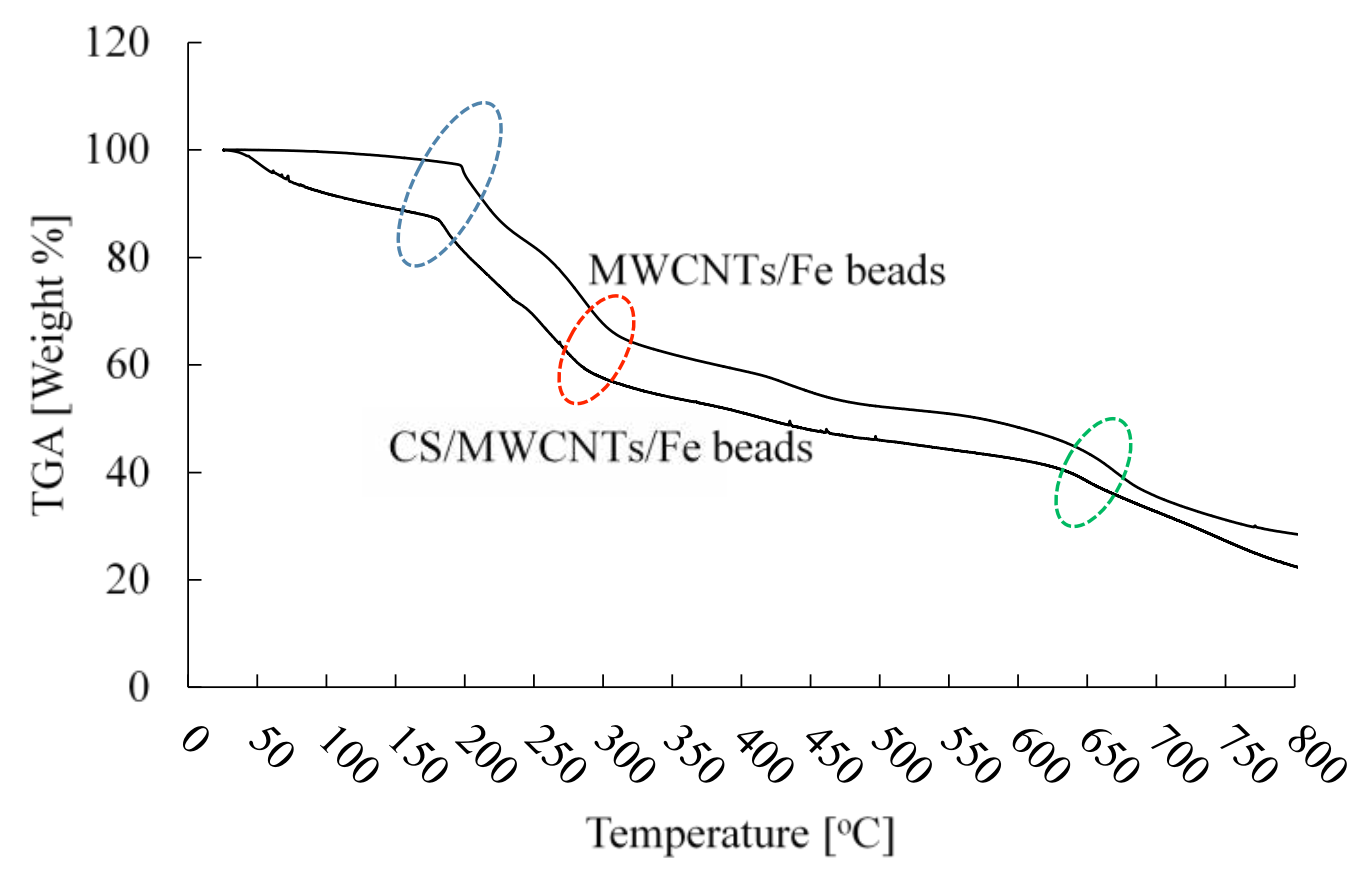




\section{Effect of $\mathrm{pH}$ on $\mathrm{Cr}(\mathrm{VI})$ Adsorption}

\section{Point zero charge}

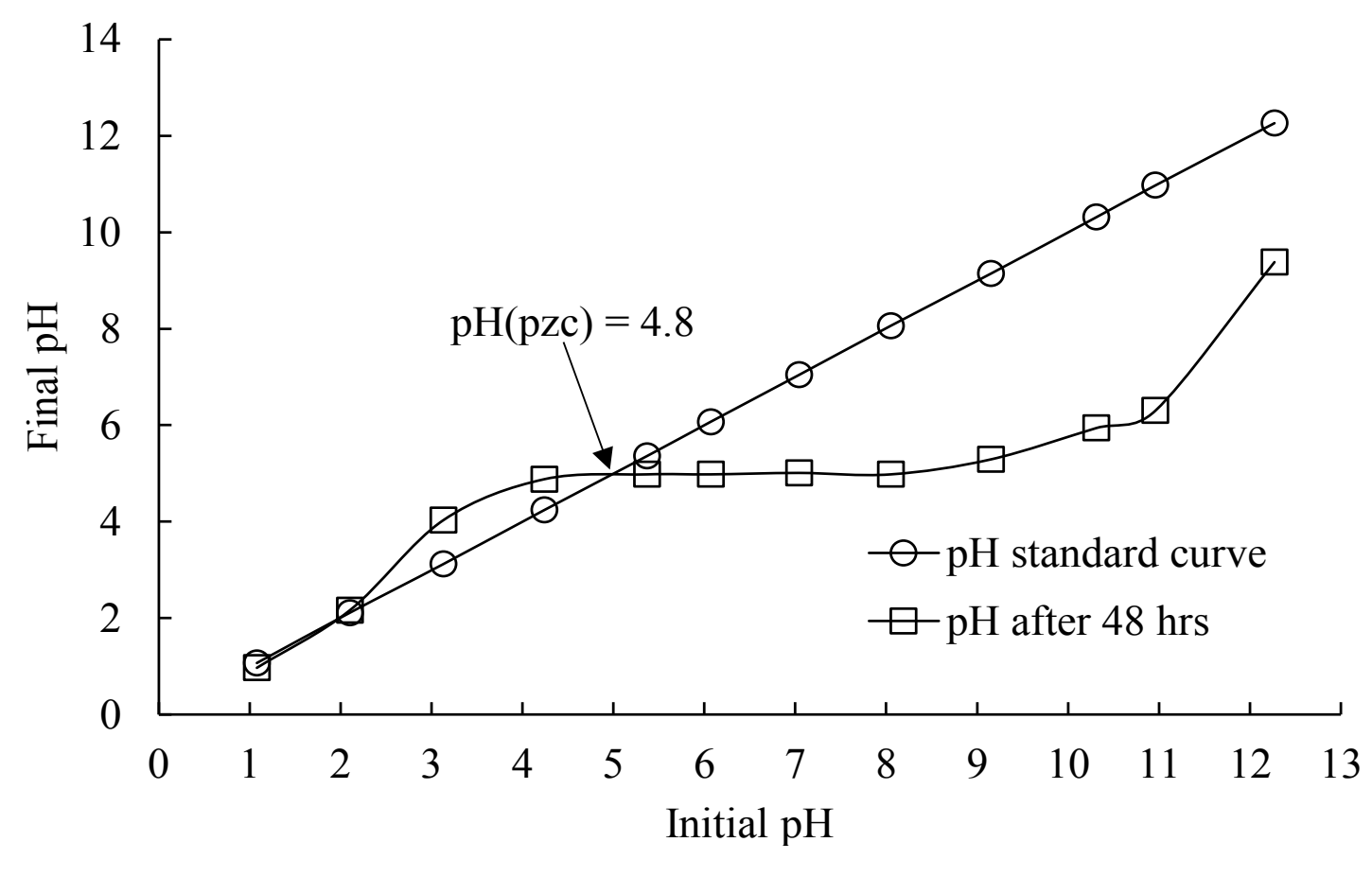

\section{Effect of pH}

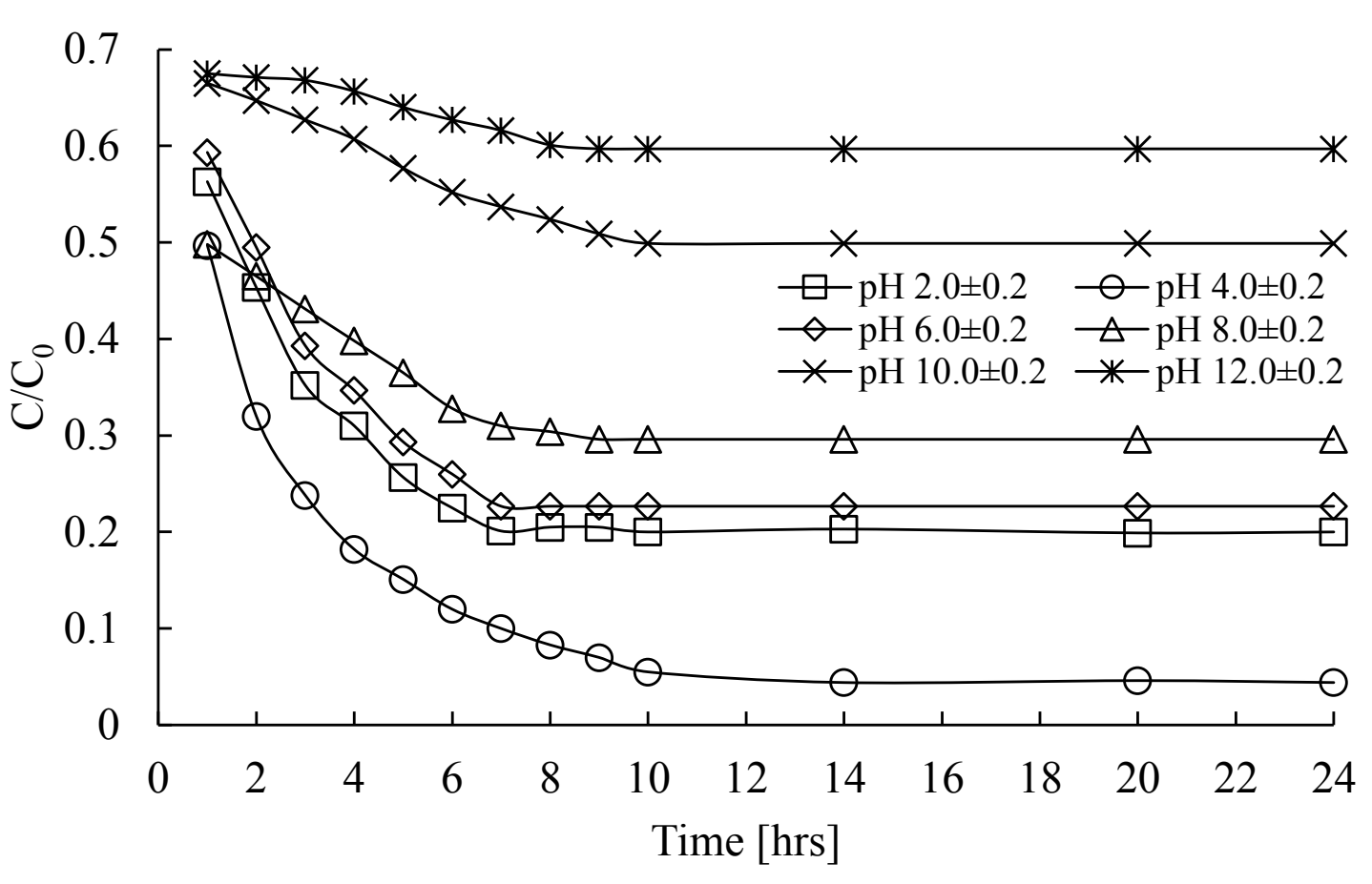

Initial $\mathrm{Cr}(\mathrm{VI})$ concentration: $10 \mathrm{mg} / \mathrm{L}$; Temperature $298 \mathrm{~K}$ 


\section{$\mathrm{Cr}$ (IV) Adsorption on CS/MWCNTs/Fe}

Effect of adsorbent dosage

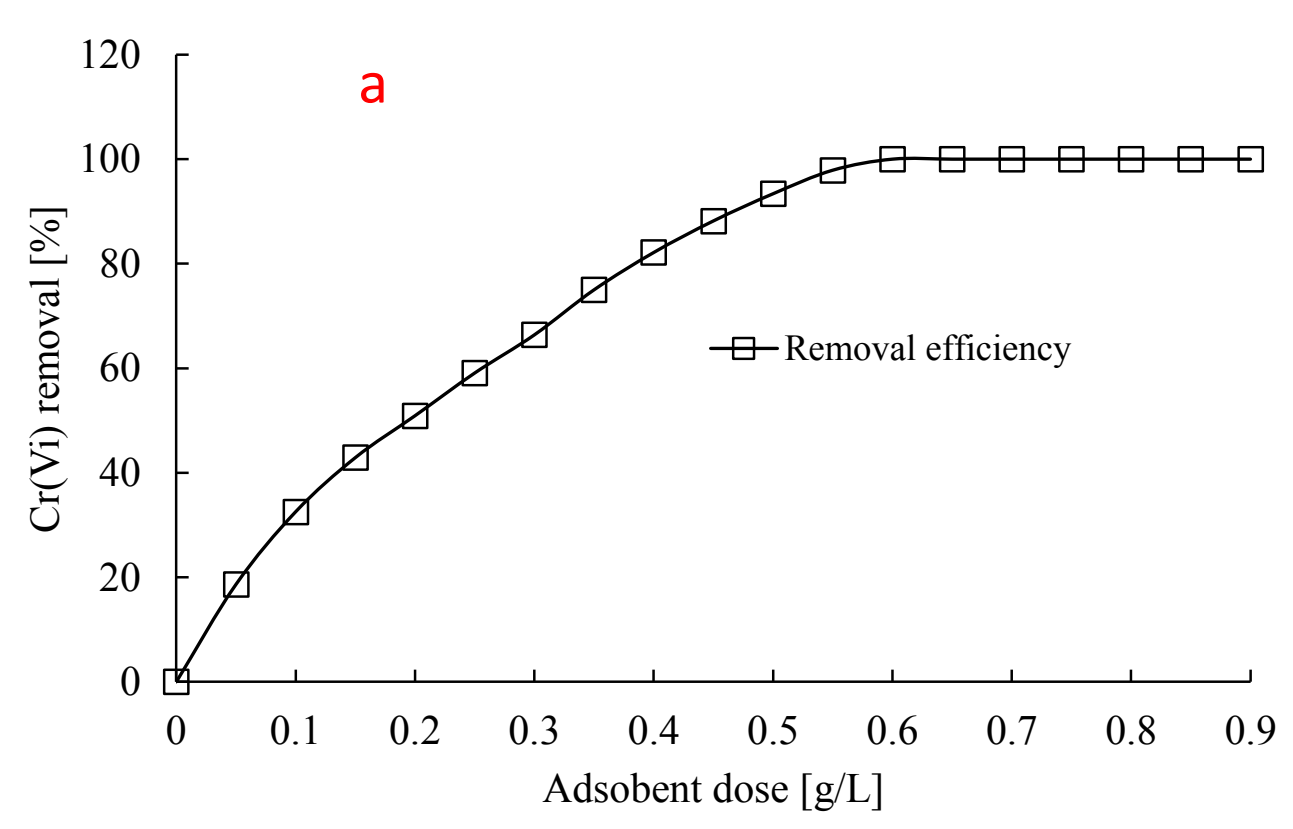

Reduction of $\mathrm{Cr}(\mathrm{VI})$ to $\mathrm{Cr}(\mathrm{III})$

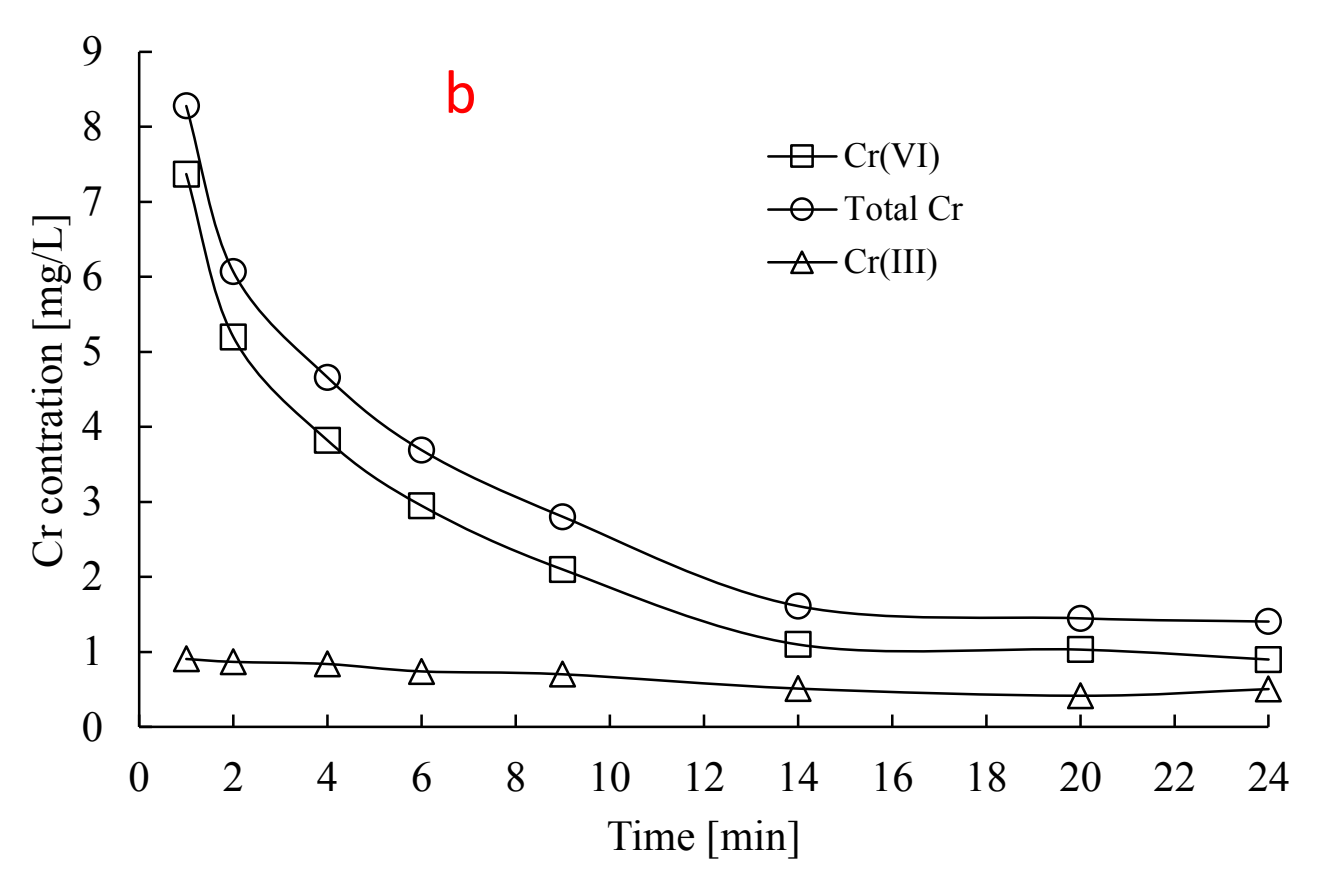

Initial $\mathrm{Cr}(\mathrm{VI})$ concentration: (a) $10 \mathrm{mg} / \mathrm{L}$, (b) $20 \mathrm{mg} / \mathrm{L}$; Adsorbent dosage: $0.5 \mathrm{mg} / \mathrm{L}$; Temperature $298 \mathrm{~K}, \mathrm{pH}: 4.0 \pm 0.2$ 


\section{Cr(IV) Removal with Different Adsorbents}

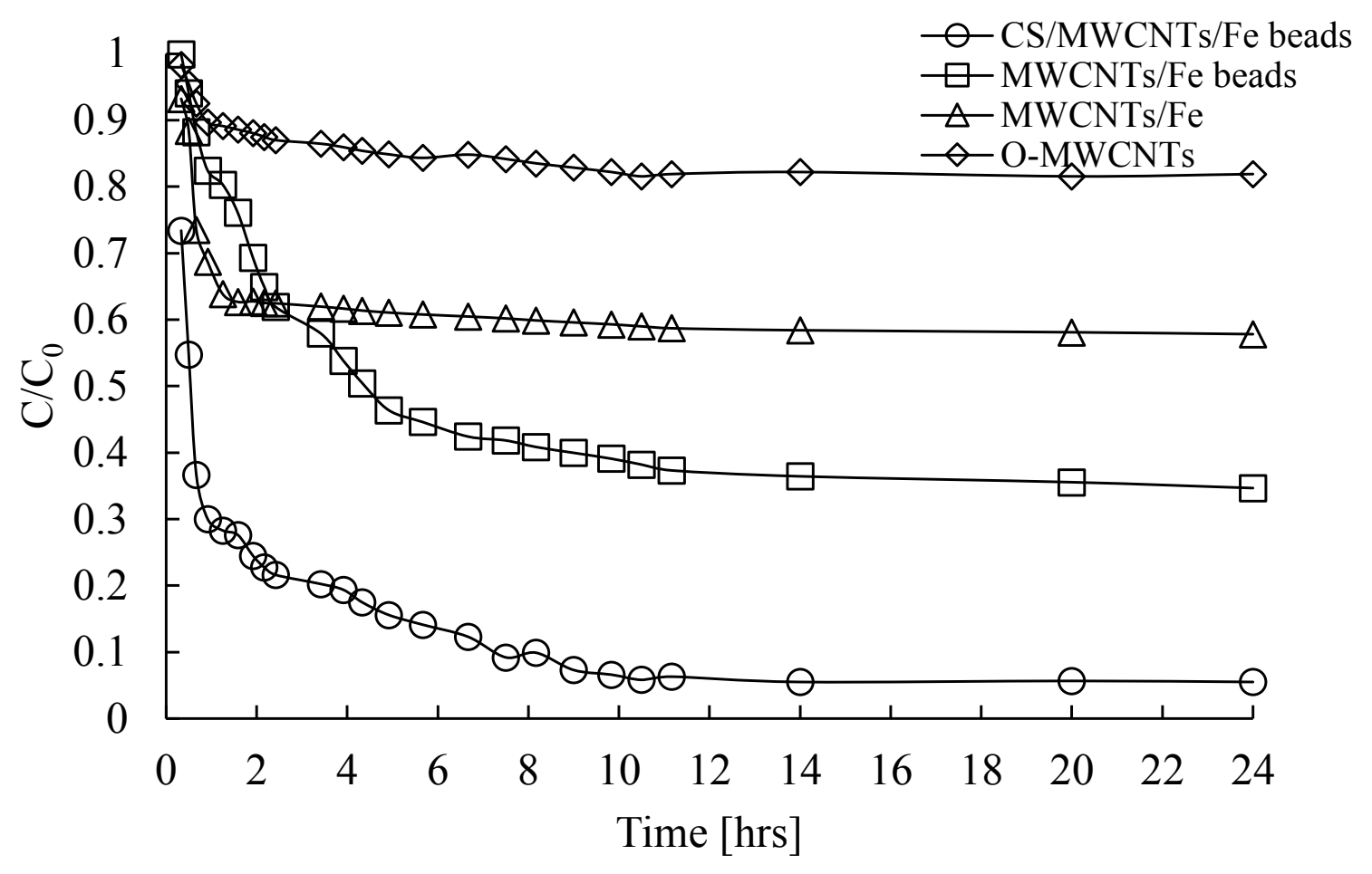

Initial $\mathrm{Cr}(\mathrm{VI})$ concentration: $10 \mathrm{mg} / \mathrm{L}$; Adsorbent dosage: $0.5 \mathrm{mg} / \mathrm{L}$; Temperature $298 \mathrm{~K}, \mathrm{pH}: 4.0 \pm 0.2$ 


\section{Effect of Material Composition}

Influence of different contents of CS

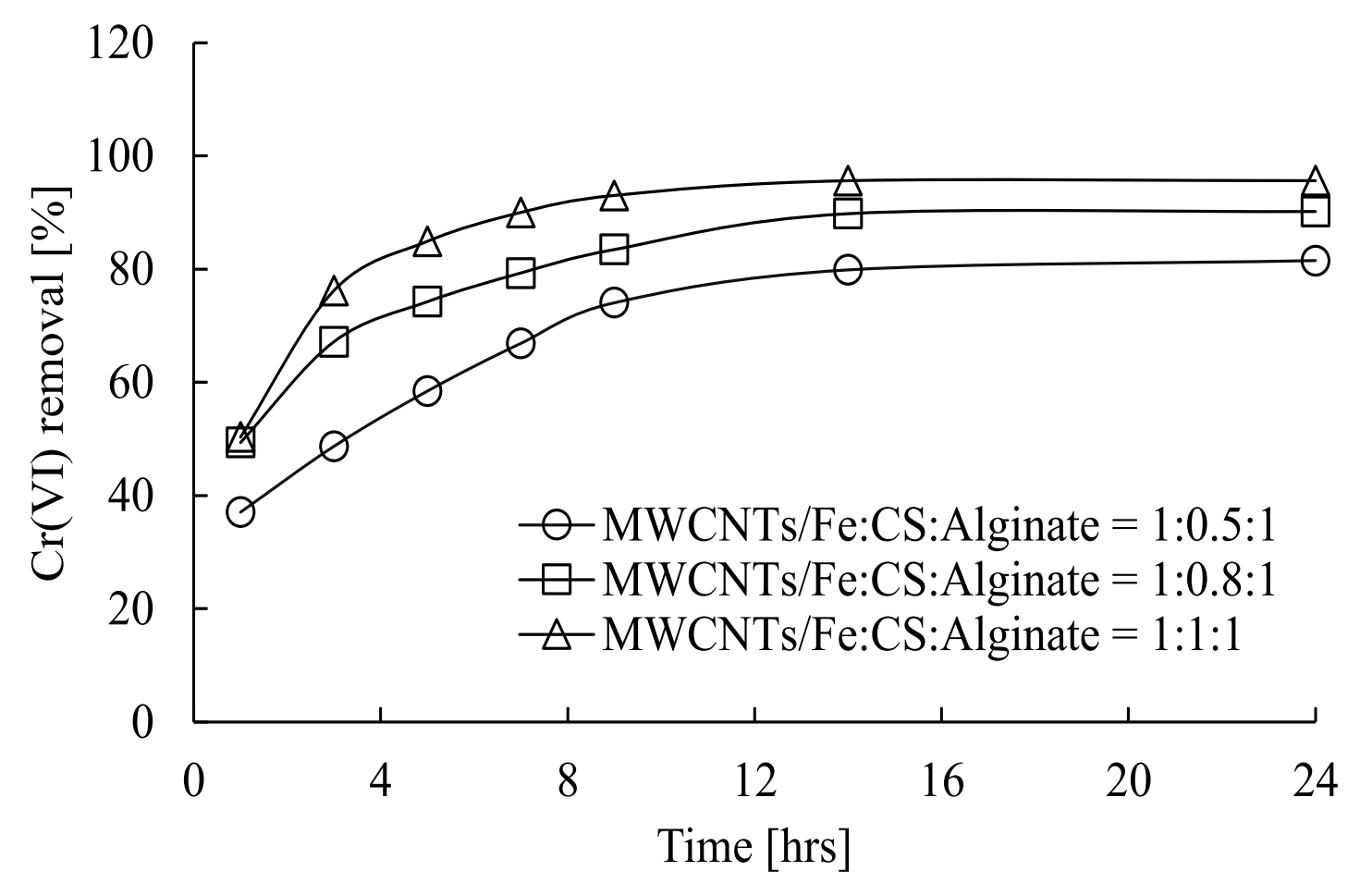

Influence of different contents of SA

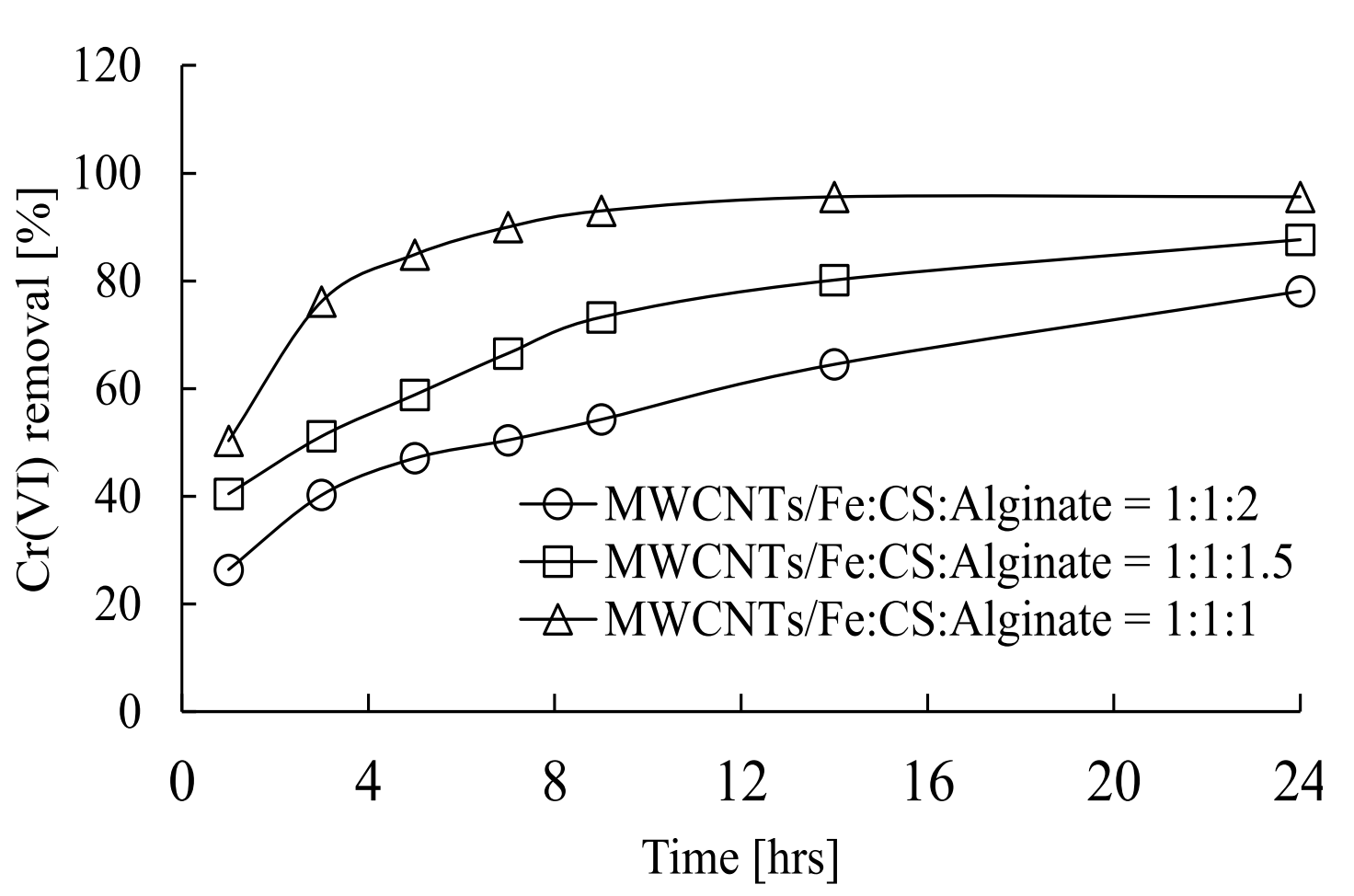

Initial $\mathrm{Cr}(\mathrm{VI})$ concentration: $10 \mathrm{mg} / \mathrm{L}$; Adsorbent dosage: $0.5 \mathrm{mg} / \mathrm{L}$; Temperature $298 \mathrm{~K}, \mathrm{pH}: 4.0 \pm 0.2$ 


\section{$\mathrm{Cr}(\mathrm{IV})$ Adsorptivity on CS/MWCNTs/Fe}

Effect of initial concentration

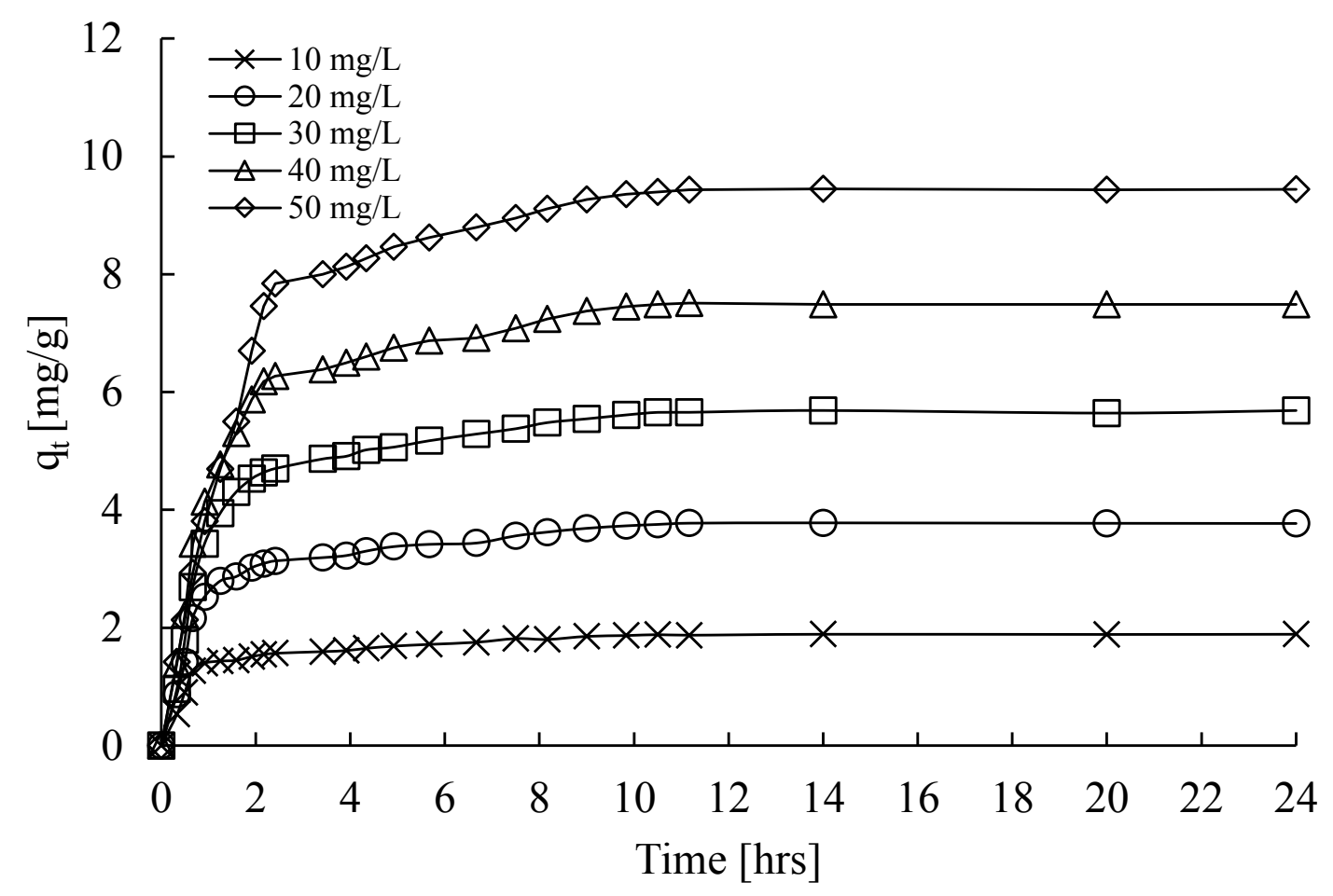

\section{Effect of temperature}

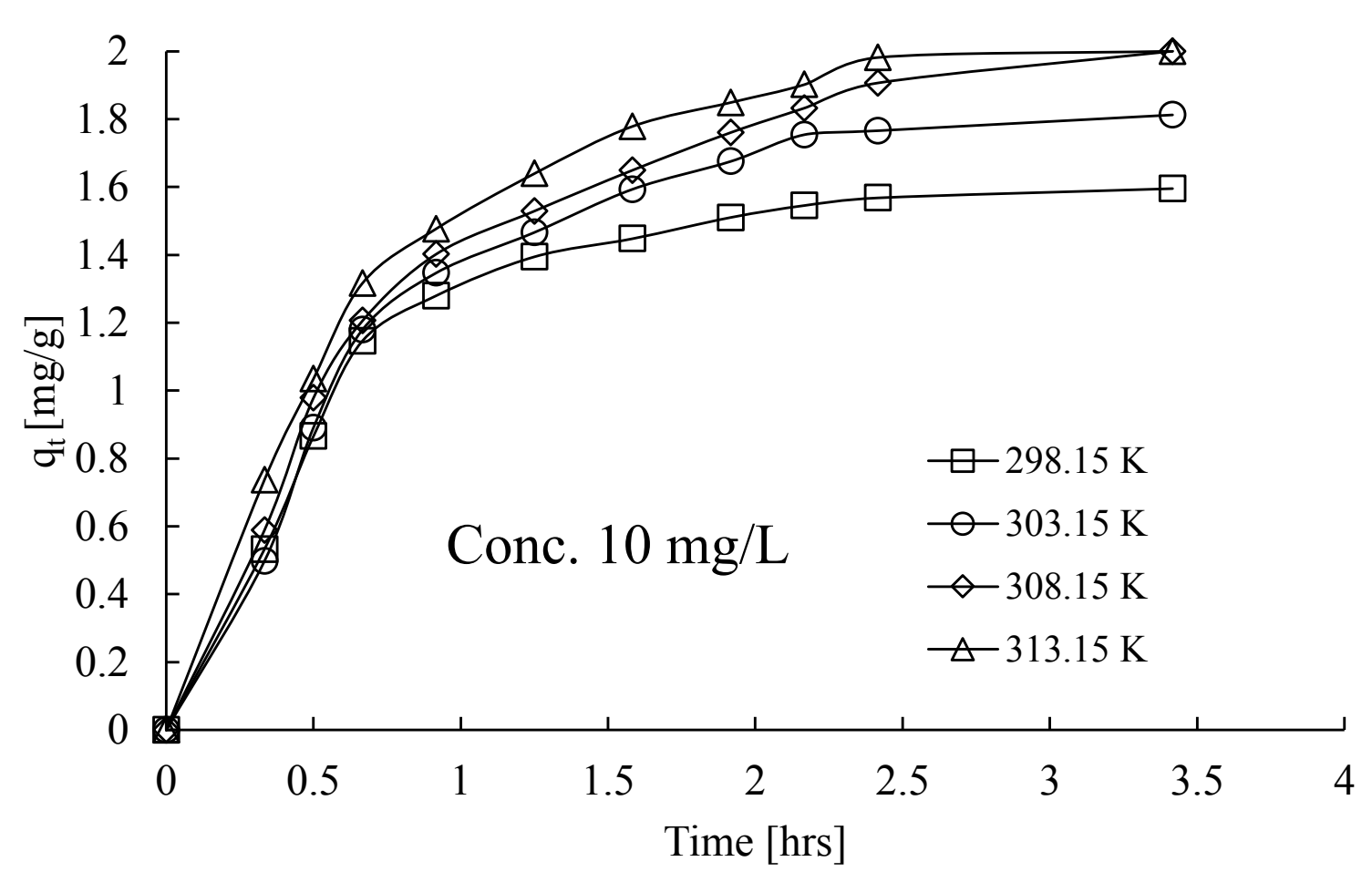

Adsorbent dosage: $0.5 \mathrm{mg} / \mathrm{L}$; Temperature $298 \mathrm{~K}, \mathrm{pH}: 4.0 \pm 0.2$ 


\section{Isotherms Study}

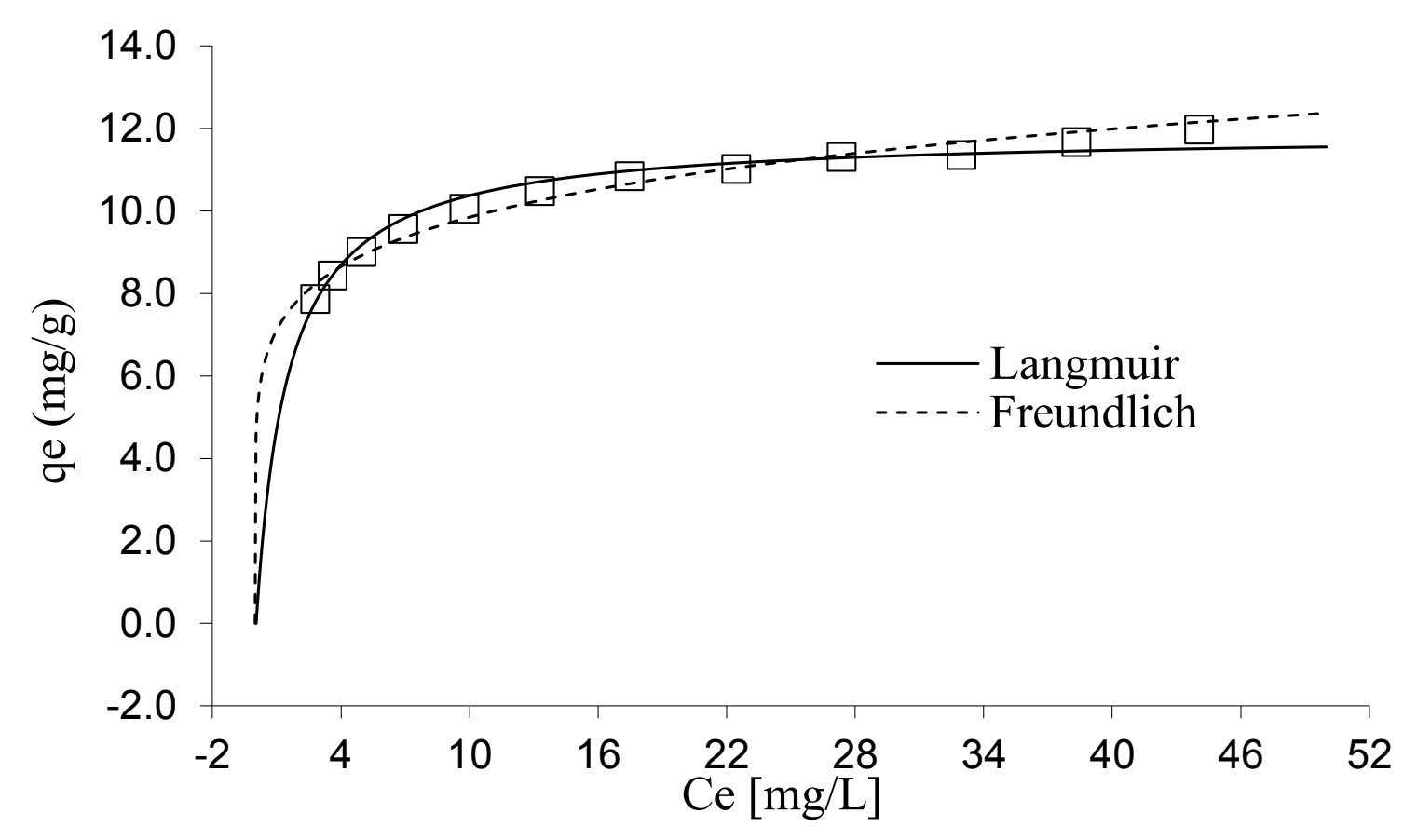

\begin{tabular}{|c|l|l|}
\hline Isotherm & \multicolumn{1}{|c|}{ Parameters } & \multicolumn{1}{c|}{ Values } \\
\hline \multirow{4}{*}{ Langmuir } & $(\mathrm{L} / \mathrm{mg})$ & 0.687 \\
\cline { 2 - 3 } & $(\mathrm{mg} / \mathrm{g})$ & 11.89 \\
\cline { 2 - 3 } & $\mathrm{b}(\mathrm{L} / \mathrm{mg})$ & 0.0841 \\
\cline { 2 - 3 } & & $0.343-0.032$ \\
\cline { 2 - 3 } & $\mathrm{R}^{2}$ & 0.975 \\
\hline \multirow{4}{*}{ Freundlich } & $1 / \mathrm{n}$ & 0.142 \\
\cline { 2 - 3 } & $\mathrm{K}_{\mathrm{F}}(\mathrm{mg} / \mathrm{L})$ & 7.101 \\
\cline { 2 - 3 } & $\mathrm{R}^{2}$ & 0.971 \\
\hline
\end{tabular}

Initial $\mathrm{Cr}(\mathrm{VI})$ concentration: $50 \mathrm{mg} / \mathrm{L}$; Adsorbent dosage: $0.5 \mathrm{mg} / \mathrm{L}$; Temperature $298 \mathrm{~K}, \mathrm{pH}: 4.0 \pm 0.2$ 


\section{Kinetic study}
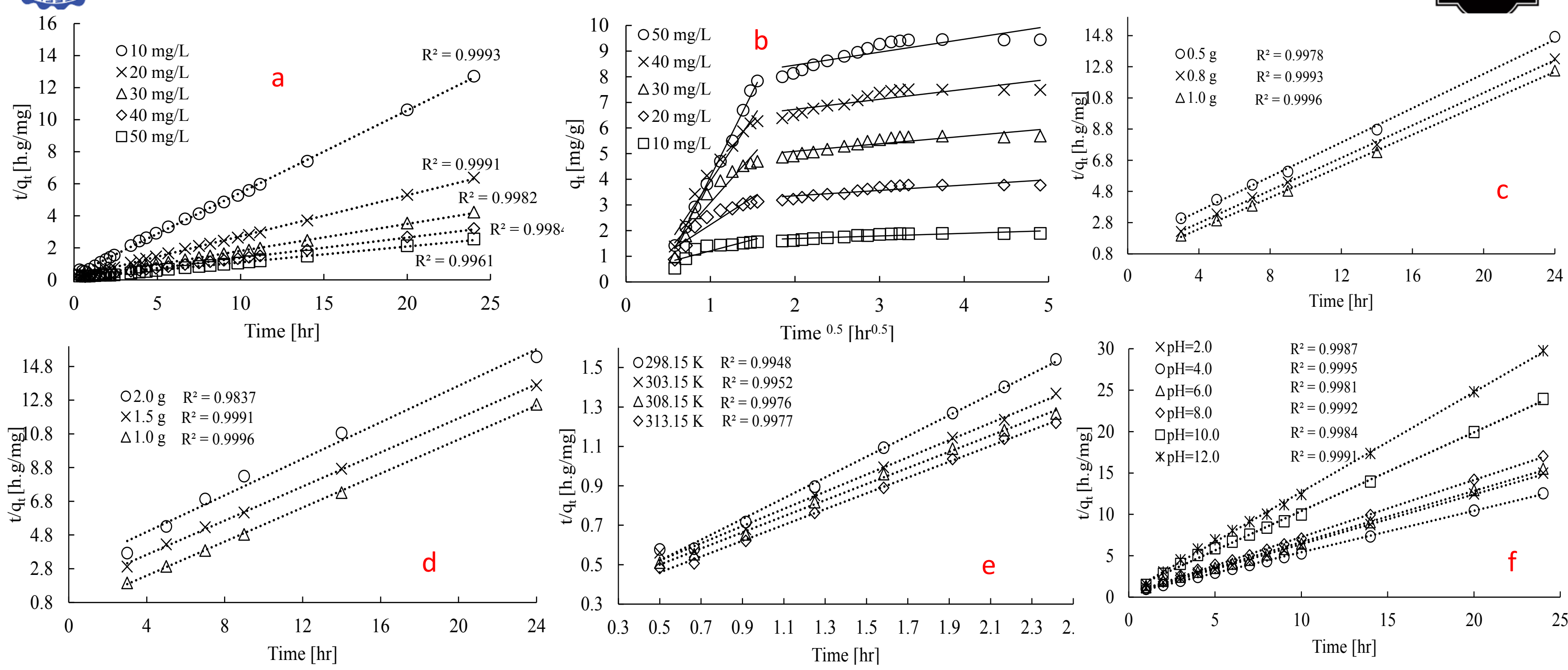

Pseudo $2^{\text {nd }}$-order: (a) initial concentration, (b) initial concentration (intra-particle diffusion), (c) contents of CS, (d) contents of SA, (e) temperature, (f) $\mathrm{pH}$ 


\section{Stability/Recyclability}

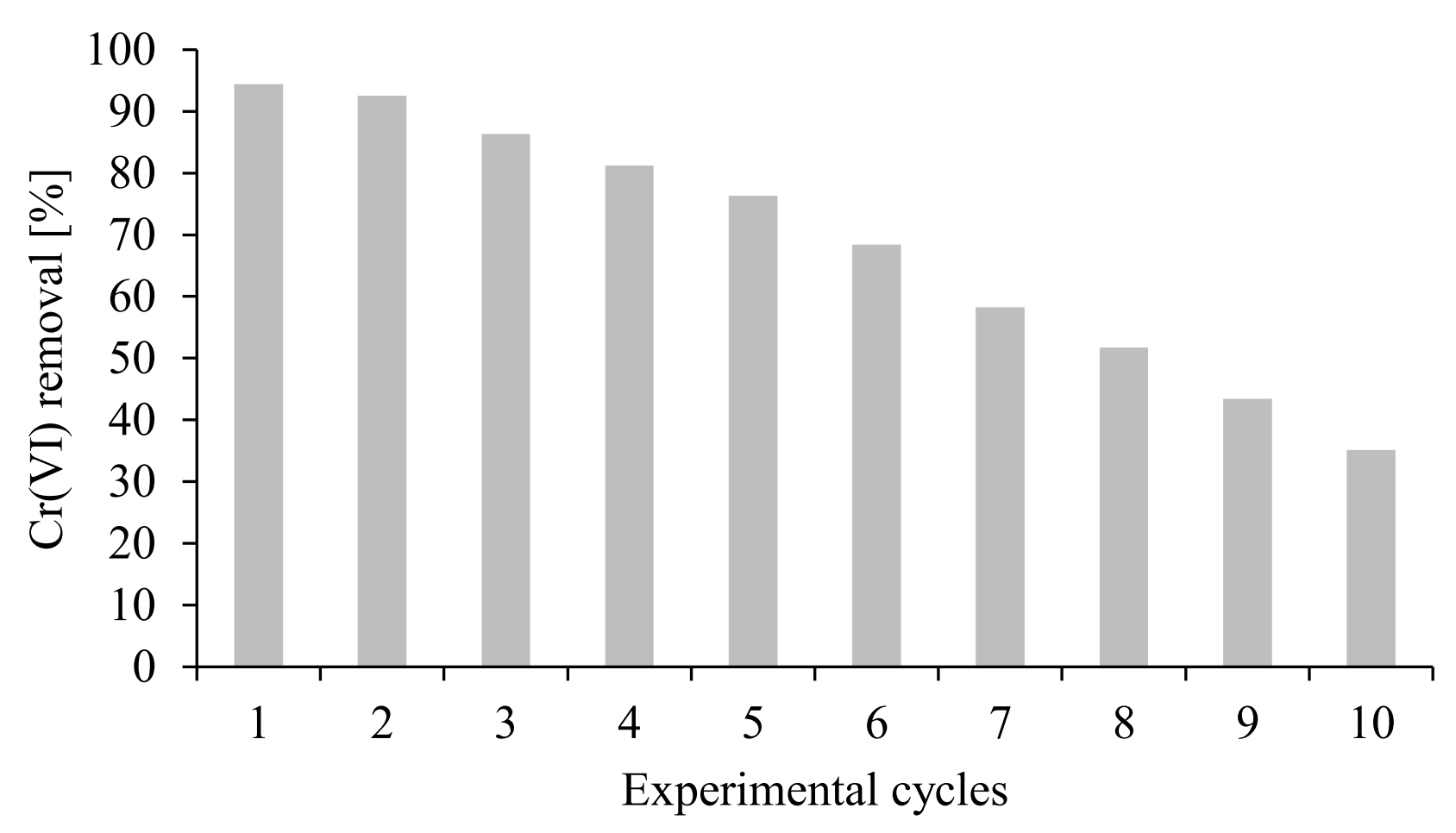

Initial $\mathrm{Cr}(\mathrm{VI})$ concentration: $10 \mathrm{mg} / \mathrm{L}$; Adsorbent dosage: $0.5 \mathrm{mg} / \mathrm{L}$; Temperature $298 \mathrm{~K}, \mathrm{pH}: 4.0 \pm 0.2$ 


\section{Summary}

- A composite adsorption material consisting of MWCNTs, chitosan, iron particles, all encapsulated as alginated beads, were successfully synthesized.

- The composite beads demonstrated a high $\mathrm{Cr}(\mathrm{VI})$ removal performance with an optimized chitosan and alginate contents. The adsorption kinetics followed closely with pseudo second order model, with a Langmuir maximum adsorption capacity of $11.74 \mathrm{mg} /$ g.

- The observation of $\mathrm{Cr}$ (III) validated the hypothesis that the addition of iron particles were capable of facilitating electron transfer from $\mathrm{Cr}(\mathrm{VI})$.

- The beads showed up to $76 \%$ of $\mathrm{Cr}(\mathrm{VI})$ removal efficiency after fives cycles of adsorption 


\section{Thank you!}

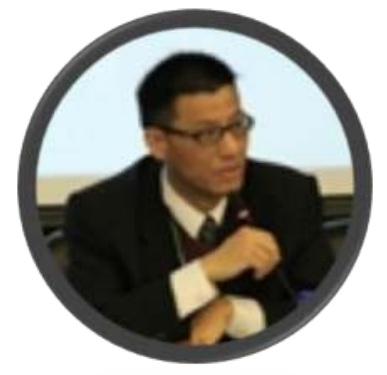

Contact: Walter Den, Ph.D. (walter.den@tamusa.edu) Professor, Water Resources Science \& Technology Department of Science and Mathematics Texas A\&M University-San Antonio ResearchGate LinkedIn Google Scholars

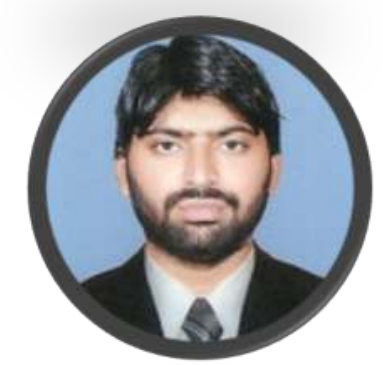

Mian M. A. Aslam, Ph.D. Candidate

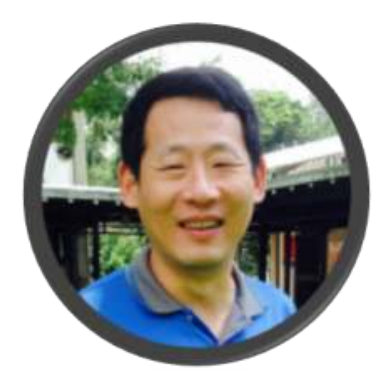

Hsion-Wen Kuo (hwkuo@thu.edu.tw) Associate Professor, Dept. of Environmental Science \& Engineering Taichung City, Taiwan R.O.C. 